KIDNEY CANCER

\title{
Tracking and therapy selection using ctDNA
}

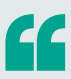

This is the

largest

assessment of

ctDNA profiles

in $\mathrm{MRCC}$

patients to

date
Despite the approval of various targeted therapies for metastatic renal cell carcinoma (RCC) over the past decade, biomarkers for allocating treatment to specific patients are lacking. The Cancer Genome Atlas (TCGA) project has enabled a deeper understanding of the genomic profile of RCC, but these data were generated from early-stage cancer, and might not translate to personalized therapy in a disease that evolves through first-line and later therapies. Thus, a biomarker that can enable longitudinal assessment of cancer genomics might be particularly valuable in RCC and enable personalization of therapy at different treatment stages. Circulating tumour DNA (ctDNA) collected from blood samples might be useful in this regard, and could be used to track tumour genomics and select next-line therapies if disease progresses.

Now, data from a recently published paper in European Urology show that the ctDNA profile in patients with metastatic RCC (mRCC) evolves with treatment. Pal and colleagues collected data from 220 patients with $\mathrm{mRCC}$ whose ctDNA profile was obtained using a platform of 73 cancer-related genes. Histological subtype was identified in 124 patients; 89 were clear cell (cc) tumours, 14 papillary, eight sarcomatoid, six chromophobe, four mixed, two undifferentiated, and one collecting duct. Multiple ctDNA samples had been collected at different time points for 17 patients, and treatment-related data regarding first-line or later-line therapies was available for 99 samples.

Genomic alterations were detected in $79 \%$ of patients overall (median 1 per patient), most frequently TP53 (35\%), VHL (23\%), EGFR (17\%), NF1 (16\%), and ARID1A (12\%). Higher frequencies of TP53 and NF1 alterations were observed in patients with sarcomatoid and chromophobe tumours, but these data did not reach statistical significance.
Although the median number of genomic alterations in first-line samples and later-line samples was similar, disparities were seen in specific genes: TP53 was altered in $49 \%$ of later-line patients versus $24 \%$ in first-line, NF1 in $20 \%$ versus $3 \%, \mathrm{VHL}$ in $29 \%$ versus $18 \%$, EGFR in $15 \%$ versus $8 \%$, and PIK $3 C A$ in $10 \%$ versus $5 \%$. Limiting of these data to patients who had received VEGF-targeted therapies only uncovered even greater disparities between later-line and first-line samples: $64 \%$ versus $31 \%$ in TP53, $29 \%$ versus $4 \%$ in NF1, and $29 \%$ versus $8 \%$ in PIK3CA.

This is the largest assessment of ctDNA profiles in $\mathrm{mRCC}$ patients to date. Lead author Sumanta Pal told Nature Reviews Urology: "Our work provides key biological insights; as patients progress from first line to salvage treatment, key changes in tumour genomics might modulate resistance. For example, the surge in TP53 suggests that this moiety might play a role in resistance."

Annette Fenner

ORIGINAL ARTICLE Pal, S. K. et al. Evolution of circulating tumor DNA profile from first-line to subsequent therapy in metastatic renal cell carcinoma. Eur. Urol. http://dx.doi.org/10.1016/ j.eururo.2017.03.046 (2017) 